\title{
ARTICLE
}

\section{MCNP neutron streaming investigations from the reactor core to regions outside the reactor pressure vessel for a Swiss PWR}

\author{
Ben Volmert $^{\mathrm{a}^{*}}$, Elena Tamaseviciute ${ }^{\mathrm{b}}$, Manuel Pantelias ${ }^{\mathrm{a}, \mathrm{b}}$, Pavol Zvoncek $^{\mathrm{b}}$ and Beat Bitterli ${ }^{\mathrm{c}}$ \\ ${ }^{a}$ National Cooperative for the Disposal of Radioactive Waste (Nagra), 5430 Wettingen, Switzerland; ${ }^{b}$ Nuclear Energy Systems Lab, \\ ETH Zurich, 8092 Zurich, Switzerland; ' Kernkraftwerk Goesgen-Daeniken AG, 4658 Daeniken, Switzerland
}

The Swiss National Cooperative for the Disposal of Radioactive Waste, partly in collaboration with ETH Zurich and the Swiss NPP utilities, has developed MCNP NPP models for neutron activation analysis and NPP decommissioning studies. These models exhibit mainly rotational symmetry and are bound to the reactor pit/drywell area. In this paper, the PWR Goesgen model is chosen as a reference model for further investigation and extended to incorporate detailed reactor pressure vessel support structures, cooling pipes/penetrations and farther ex-reactor pressure vessel areas such as the adjacent steam generator or cooling pump compartment as well as the layout of the basin area above the reactor pressure vessel. The impact of these azimuthal asymmetries on the ex-reactor pressure vessel neutron distribution is quantified and the variance reduction methodology used is outlined. Finally, a description of the in-situ foil irradiation campaign initiated in collaboration with the Swiss NPP Goesgen for the purpose of future model validation is presented.

Keywords: MCNP; NPP; decommissioning; streaming; activation; neutron flux distribution; validation

\section{Introduction}

In the context of the periodic Swiss NPP decommissioning studies, MCNP (version 5.1.60, standard cross section libraries) NPP models for each of the Swiss NPPs have been developed for the purpose of component activation calculations [1].

More specifically, mostly cylindrically symmetric MCNP models extending to the reactor pit/bio-shield (for PWRs) and into the drywell (for BWRs) were developed in order to calculate the neutron spectra within the main "in-reactor pressure vessel (in-RPV)" and ex-RPV components such as core support plates, barrel, baffle, jet pumps area, RPV, bio-shield, additional concrete structures and its rebar, etc. The resulting spectra filling a corresponding activation zone map are used for the ensuing activation calculations.

The results of these theoretical investigations supported by the radiological characterization of RPV samples from the already dismantled German BWR Gundremmingen-A unit and other in-situ neutron measurements show that neutron streaming through gaps, mainly between the RPV and the bio-shield, plays a significant role in the activation pattern of a NPP [2].

In order to further examine the impact of neutron streaming beyond the previous NPP model boundaries, the MCNP model of the "Goesgen PWR (KKG)" is extended to include the three loops of cooling pipes, an adjacent reference side room representing a typical steam generator/cooling pump compartment and an improved layout of the basin area above the RPV head (see Figure 1) [3]. The calculated neutron flux distribution for this extended KKG model is discussed in detail in the following section.

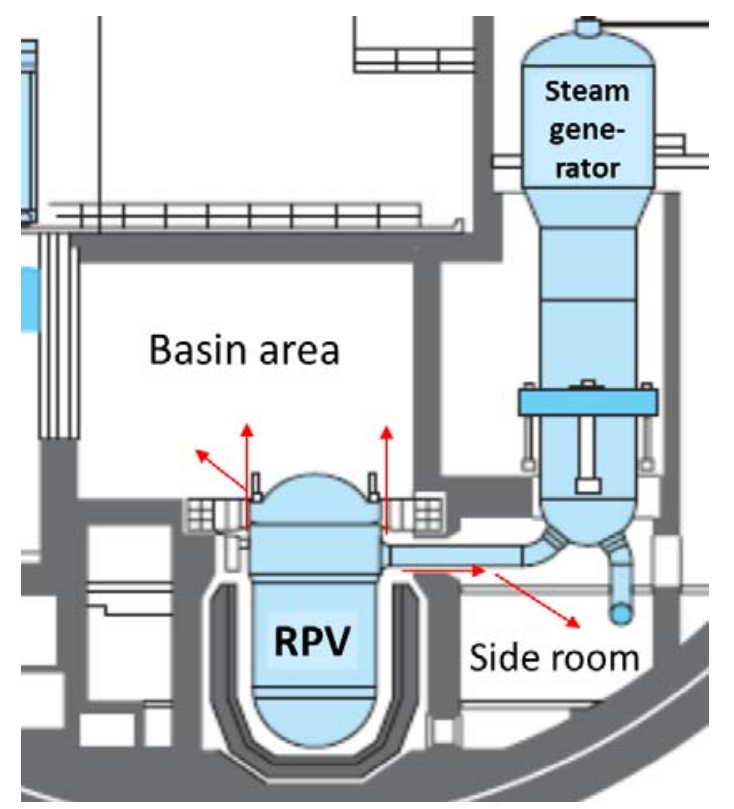

Figure 1. Example of ex-RPV neutron streaming pathways.

*Corresponding author. Email: ben.volmert@nagra.ch 
In order to validate the calculations, an in-situ foil irradiation campaign with KKG was initiated for the 2011-2012 power cycle to deliver 3-group neutron flux values (ORIGEN-S-boundaries at $0.625 \mathrm{eV}$ and $1 \mathrm{MeV}$ ) at representative locations outside the RPV. The final results of this campaign are expected to be available in spring 2013. A short description of the foil irradiation campaign together with some first results will be presented in section 3 of this paper.

\section{Extended KKG MCNP model}

\subsection{Geometry and materials}

The MCNP modeling of the KKG PWR is based on technical drawings of the plant. Material compositions and densities used are based on plant-specific information or, when not available, on literature data. Heterogeneous material distributions are mostly homogenized (reinforcement bars (rebar) in concrete, control rod drive area, thermal insulation around the RPV etc.), except for components of importance to the ex-vessel flux distribution such as internals like the core barrel, the thermal shield and the RPV. The six cooling pipes and the corresponding bio-shield penetrations leading outwards are explicitly modeled, together with the components surrounding the RPV head and the individual RPV support structures confining the gap cross-section for the streaming path into the basin area (see Figure 2).

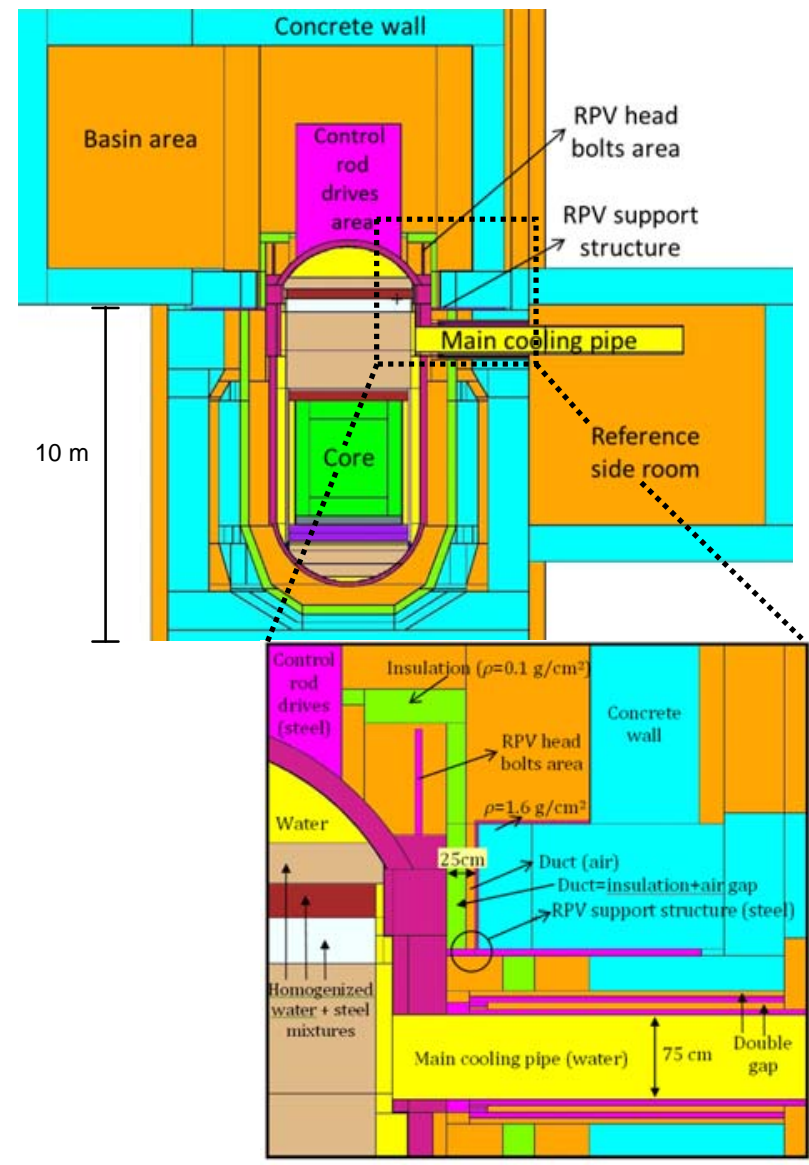

Figure 2. MCNP KKG model (zoom: cooling pipe section).

\subsection{Neutron source}

The fixed source core model is based on typical middle-of-cycle operating conditions (i.e. average thermal core total power, fuel assembly specific relative and axial power profiles, boron concentration etc.). The inner part of the core is set as inactive for computational efficiency. A more detailed description of the core source for the NPP activation calculations can be found in previous works [1].

\subsection{Variance reduction}

As it is obvious from the scale of the problem, significant "variance reduction (VR)" efforts are needed in order to ensure the statistical significance of results in areas far from the core. The combination of the thick shielding and the existence of streaming paths for the reactor results in a challenge of a deep shielding penetration and streaming problem. In fact, total fluxes within the model boundaries span over up to 15 orders of magnitude and the streaming of neutrons through the existing gaps significantly contributes to the majority of the calculated tallies.

The VR methodology implemented uses the cylindrical mesh based "weight window (ww)" generator. The mesh grid covers the whole model boundary and consists of a coarse axial and a more resolved radial segmentation. The ww parameter generation is optimized for the farthest tallies from the reactor core. For each of these target tallies an individual VR iteration sequence is performed. The typical VR iteration sequence consists of multiple runs, beginning with a reduced density run followed by an individual mixture of gradually increasing the density back to nominal values and using auxiliary tally locations (i.e. "jumping” towards the target tally). The flux distribution maps of the different target tally calculations are finally merged by a small Excel-script algorithm. This algorithm decides in cases of doubt which single mesh tally cell result is the most plausible one - a decision based mostly on the values' uncertainties. It also gives a warning to the script user if the chosen specific mesh cell value would lead to strange results between cells in its close vicinity, which could lead to an overriding of the script result and a manual correction and by the user.

The results are tallied with the help of both mesh tallies and cell based tallies (all of them are "track length estimator" types). The energy binning used, an 84-energy-group flux structure, corresponds to the interface of the activation code used [1] but, additionally, simpler 3-group fluxes are also tallied in order to facilitate the qualitative streaming analysis even with a reduced computational effort.

It is worth noting that the laborious VR method described above is indeed needed in order to produce sufficiently effective ww parameters for the lowest flux regions deep inside concrete and even far from the source (e.g. in the concrete walls of the reference side room).

Although the implemented ww based VR approach delivered the desired results, additional work is currently 


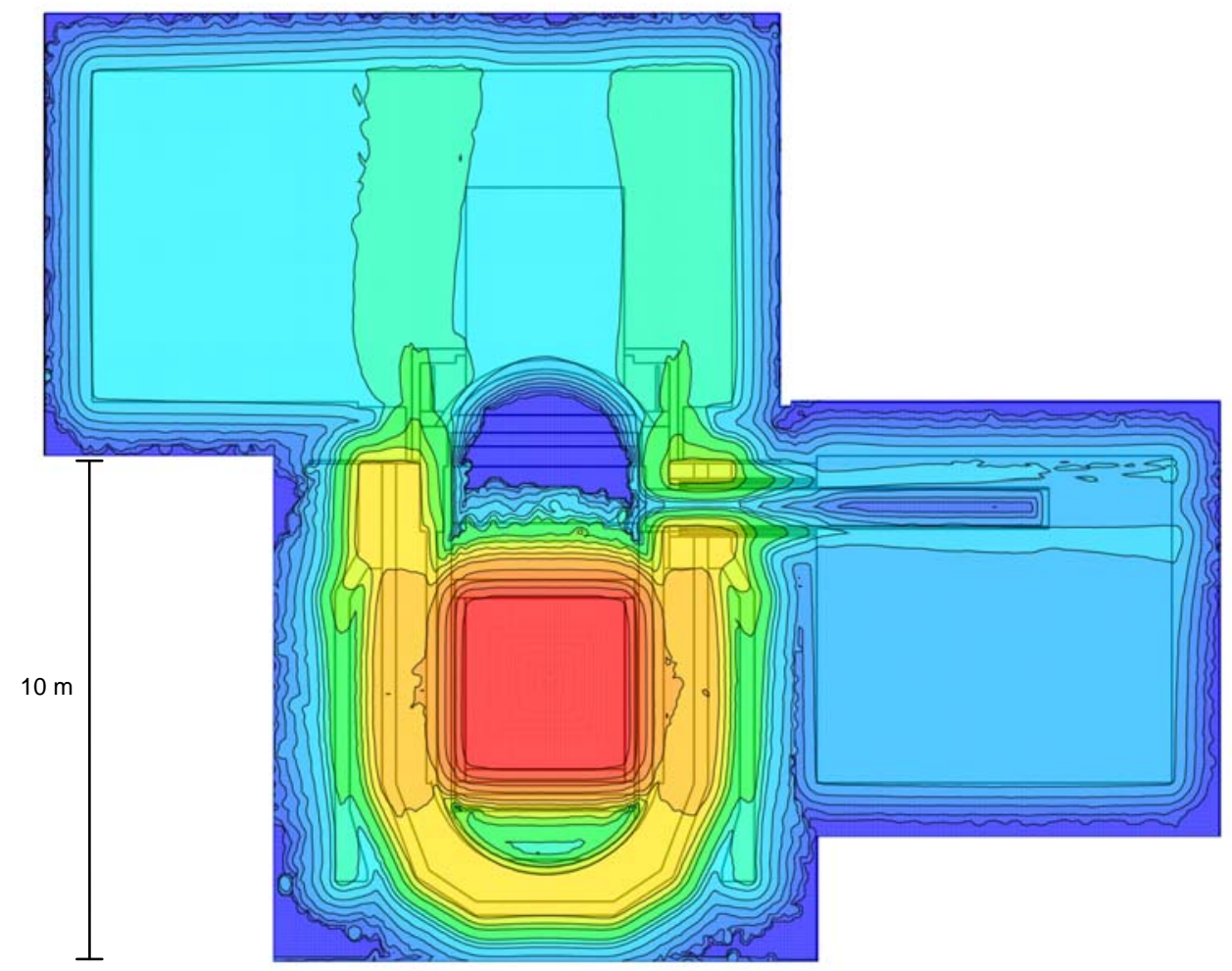

total neutron flux $\left[\mathrm{cm}^{-2} \mathrm{~s}^{-1}\right]$

$1 E+14$

$1 \mathrm{E}+13$

$1 \mathrm{E}+12$

$1 \mathrm{E}+11$

$1 \mathrm{E}+10$

$3.16 \mathrm{E}+09$

$1 \mathrm{E}+09$

$3.16 \mathrm{E}+08$

$1 \mathrm{E}+08$

$1 \mathrm{E}+07$

$3.16 \mathrm{E}+06$

$1 \mathrm{E}+06$

100000

10000

1000

100

10

Figure 3. Neutron flux distribution for the extended KKG MCNP model (for a typical middle of cycle situation).

being carried out at Nagra for the implementation of hybrid methods [4] which are expected to yield significant computational speedups and less manual engineering work.

\subsection{Neutron flux distribution}

The resulting neutron flux distribution for the extended KKG MCNP model is presented in Figure 3. The total flux distribution is tallied by a rectangular mesh tally overlaying the geometry parallel to the cooling pipe leading to the reference side room.

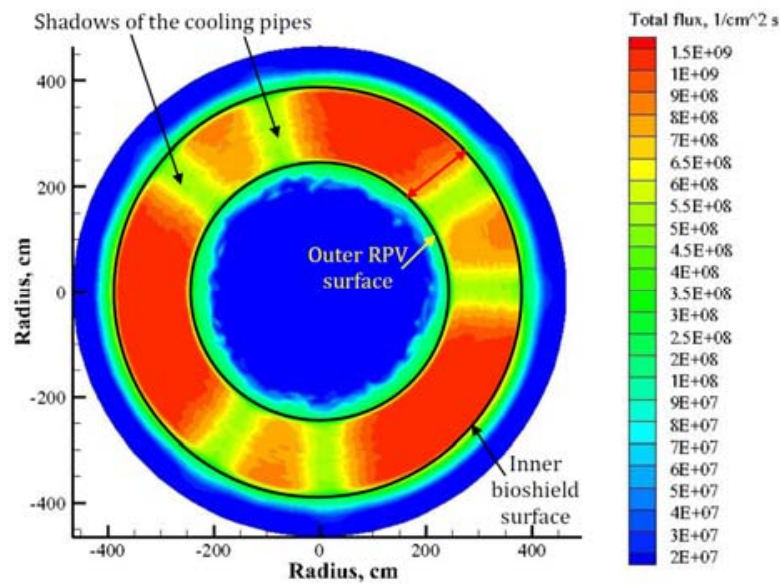

Figure 4. Neutron total flux distribution in a horizontal plane directly above the six cooling pipes.
The statistical uncertainties of the total neutron flux values of mesh tally in all figures of this paper are generally well below $10 \%$ except for most of the model boundary areas (i.e. deep inside the concrete walls), the top area inside of the RPV and some distinct narrow streaming pathways (like the exit of the cooling pipe). In these areas the significant flux uncertainty is visible by the strong fluctuations of the flux iso-lines.

The streaming of the neutrons alongside the cooling pipe ring gap into the side room and through the RPV head ring gap into the basin area can be clearly seen. Also, the flux depression inside the pipe and within the concrete walls is well demonstrated. The flux level within the side room is approximately two orders of magnitude below the level in the basin area above the RPV head.

\subsection{Shielding effect of cooling pipes}

The main cooling pipes of the RPV provide a considerable shielding effect to the neutron streaming into the reactor basin area. More specifically, the inclusion of the cooling pipe structures into the extended model decreases the effective neutron source for the basin area above by about $50 \%$.

A visual representation of the shadow effect of the cooling pipes is provided by Figure 4. The shadow effect of the cooling pipes is most prevalent for the fast neutron group (decrease of a factor of $\sim 10$ in the shadowed parts), whereas a flux reduction of about a factor of 2 is observed for the thermal and epithermal 
ranges. For the total flux, the shadow effect reduces the flux on top of the cooling pipes compared to the unobstructed pathways by around a factor of 3 (see Figure 4).

Additionally, it is worth noting that, at the axial level directly above the pipe, epithermal neutrons play the dominant role, together with fast neutrons. The thermal neutrons contribute approximately one order of magnitude less to the total flux.

\subsection{Streaming through the RPV head ring gap into the basin area}

As the neutrons stream up around the cooling pipes into the ring-like gap around the RPV head, the shadow effect of the cooling pipes diffuses. At the exit of the ring gap into the basin area, where the average total neutron flux level has dropped by a factor of $~ 20$ in comparison to the level directly above the cooling pipes, any visible shadow has mostly vanished.

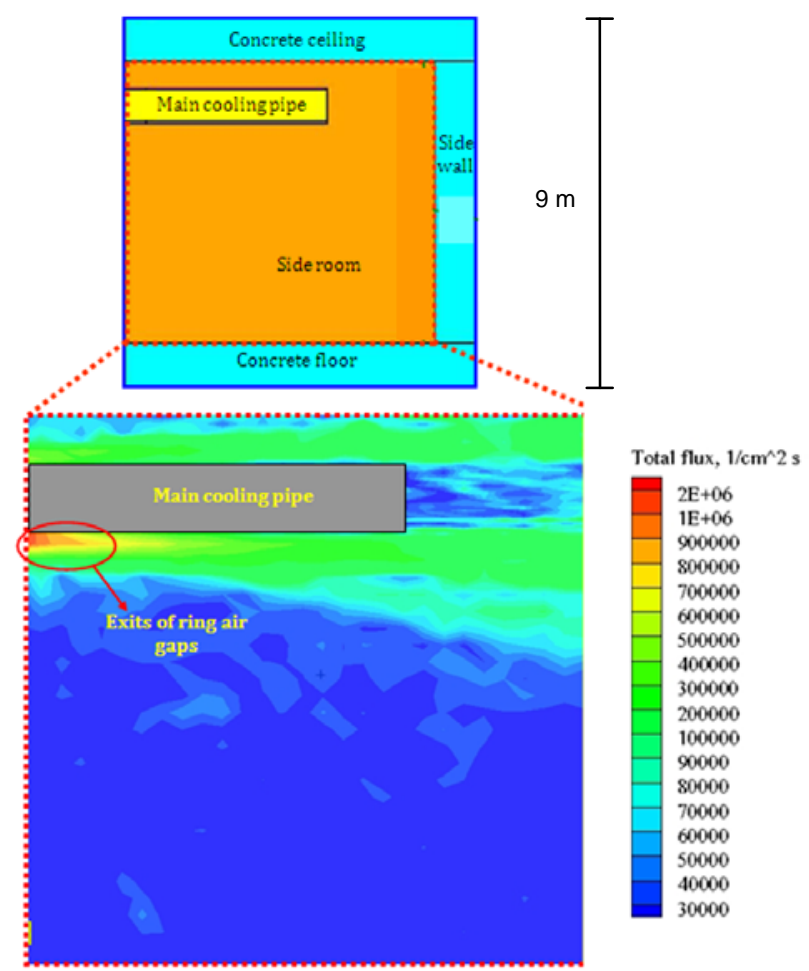

Figure 5. Neutron total flux distribution in a vertical plane parallel to the cooling pipe (reference side room).

The order of magnitude of the neutron flux within the basin area is finally reduced by a factor of $3-4$ by the inclusion of the cooling pipes and the RPV support structures into the extended KKG model, compared to the rotational symmetric model before - with direct consequences for the level and amount of activated components of the KKG basin area.

\subsection{Streaming into the reference side room}

The streaming of neutrons into the reference side room is shown in Figure 5. The neutrons stream along the double ring gap of the cooling pipe penetration through the bio-shield into the side room. Facing the gap exit there is a top-bottom as well as a right-left asymmetry in the neutron streaming profile that is caused by the scattering of neutrons at the bottom of the pipe as well as at the directly adjacent pipe before entering the gap.

Although the neutron flux level inside the side room is much lower than in the basin area, it still could lead to activity levels of side room installations and walls above the current release limit and is therefore important to take into account in any decommissioning study.

The results for the extended model shown justify the inclusion of the extended modeling features into future NPP activation studies since, in some parts of the plant, the distribution as well as the absolute levels of activity have been significantly changed compared to the results of the rotational symmetric NPP model [3].

\section{KKG/Nagra foil irradiation campaign}

Even the extended KKG model can only represent a very rough simplification of the plant itself. Furthermore, most of the installations within the side room and the basin area are omitted because of modeling and efficiency constraints. Although the extended model has been created with the purpose of representing all significant aspects for the neutron transport and streaming simulation, any validation for this study can only be done by adequate measurement of neutron fluxes and spectra within the plant itself.

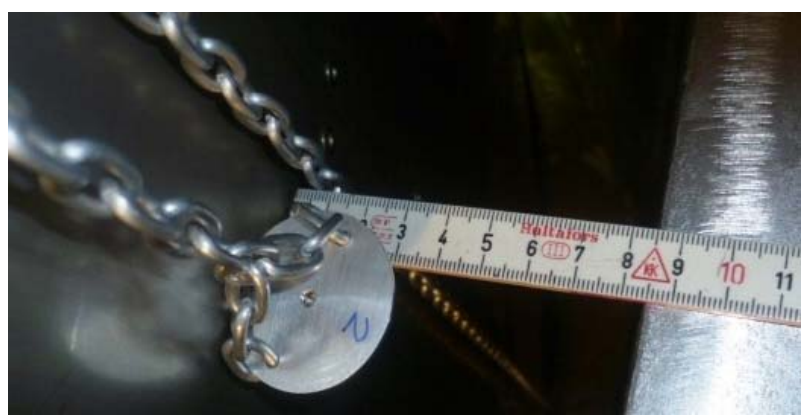

Figure 6. Typical KKG foil sample (at RPV ring gap).

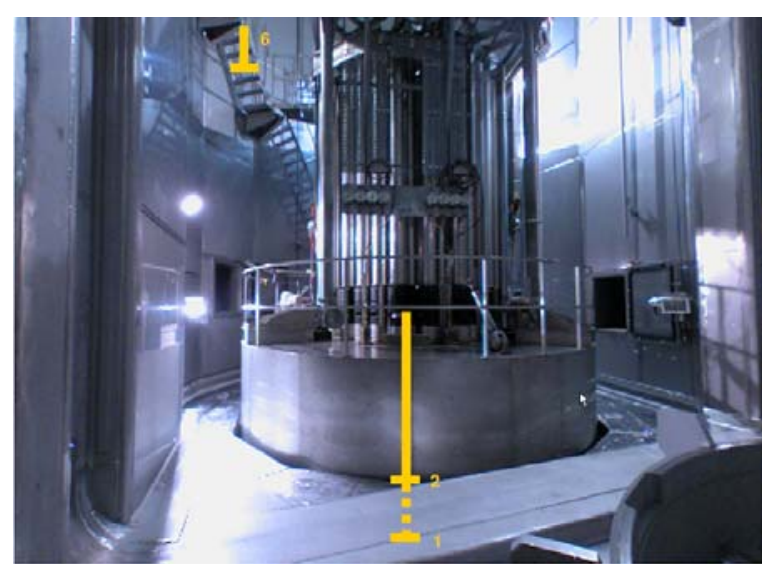

Figure 7. Two example positions where the samples were placed within the basin area. 
For this reason, a foil activation campaign was initiated during the maintenance shutdown of KKG in summer 2011 and ten samples were placed at specific locations within the basin area, the RPV ring gap and the side room (see Figures 6 to 8 ). The samples each consist of three encapsulated activation foils (Ni, Ag, Co) to analyze at least three different energy regions of the neutron flux. After one full KKG operation cycle, the samples were retrieved in summer 2012 and will now be analyzed for any spectral information representing the neutron fluxes at their locations.

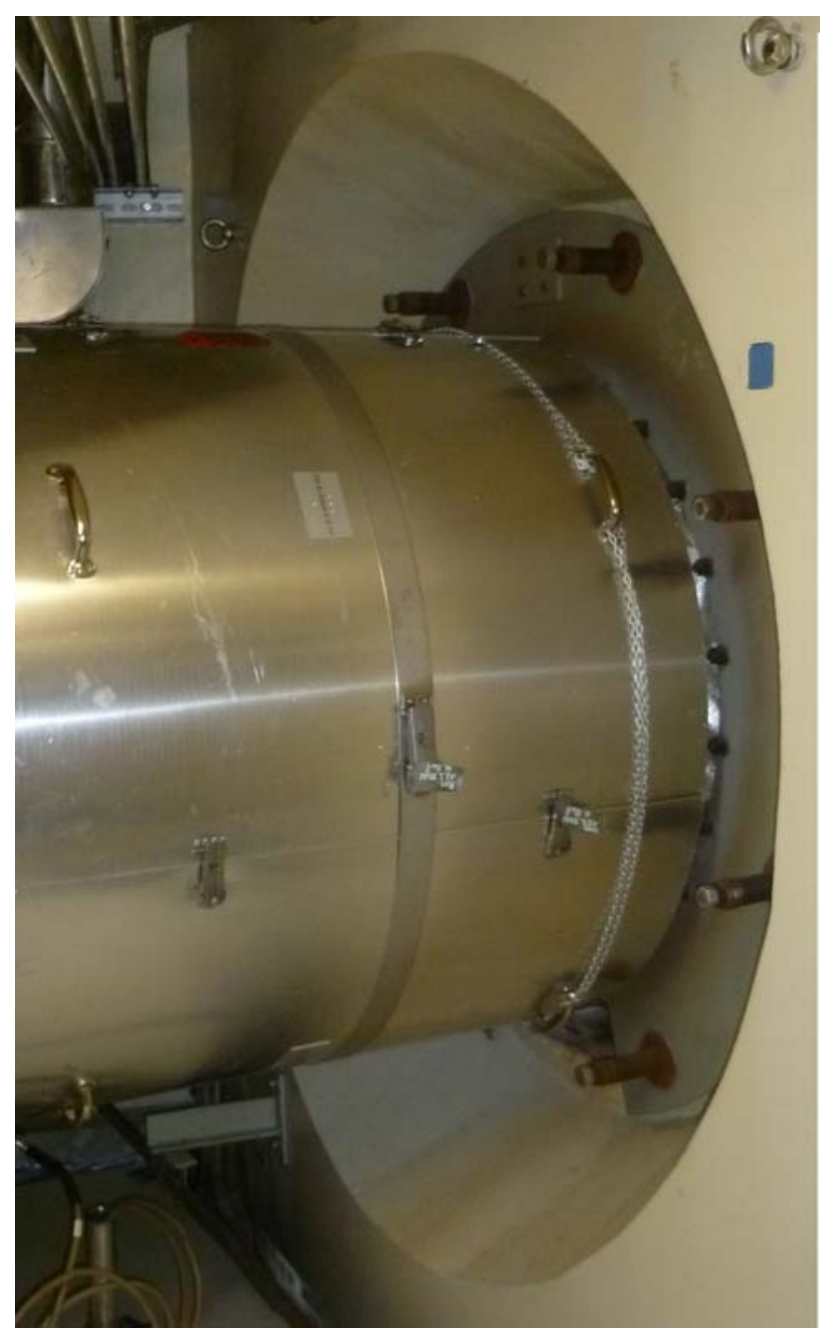

Figure 8. Cooling pipe samples with fixation chain.

A first on-site measurement by KKG personnel, which was not suited for the determination of absolute activity levels, still indicates that the activity levels of most samples match the relation of the corresponding calculated total fluxes for the extended KKG model within a factor of 2.5, often even much better. The expected analysis of the activated samples in spring 2013 by a contracted research lab will be supported by a generic in-house MCNP simulation to investigate the effects of possible sample self-shielding, neutron spectrum variations and scattering corrections by adjacent components/walls.

\section{Conclusion}

In this paper, the PWR Goesgen (KKG) MCNP model - already used for the NPP activation calculations in the context of the Swiss 2011 decommissioning study - is extended by including the 6 cooling pipes, one reference side room and a more detailed layout of the RPV head and basin area.

These extensions prove that the currently used variance reduction efforts are at their practical limit and that for further model extensions the use of a hybrid variance reduction method [4] should be pursued.

The resulting overall neutron flux distribution is significantly influenced by the new streaming pathways of the extended KKG MCNP model which in turn leads to different activation patterns for KKG components and building structures.

The on-going work towards validation of the methodology by an NPP sample irradiation campaign is outlined and a final analysis report is expected in 2013 first on-site measurements already show encouraging results comparing the measured sample activities with the total neutron flux calculated.

\section{References}

[1] M. Pantelias, B. Volmert, S. Caruso, P. Zvoncek, B. Bitterli, E. Neukaeter, W. Nissen, G. Ledergerber, and R. Vielma, MCNP modeling of the Swiss LWRs for the calculation of the in- and ex-vessel neutron flux distributions, Proc. PHYSOR 2012 Advances in Reactor Physics, Knoxville, Tennessee, USA, April 15-20, (2012). [CD-ROM]

[2] M. Pantelias, Ch. Lierse von Gostomski, U. Krumpholz, B. Volmert, R. Artinger and M. Filss, Reactor pressure vessel activation: RPV sample analysis and Monte Carlo simulations, Proc. KONTEC 2011, Dresden, Germany, April 5-8, (2011). [CD-ROM]

[3] E. Tamaseviciute, Neutron Streaming Investigations Outside the RPV of the Goesgen Nuclear Power Plant with the Monte Carlo Method, M.Sc. Thesis, ETH Zurich, (2011).

[4] J. C. Wagner, D. E. Peplow, S. W. Mosher and T. M. Evans, Review of hybrid (deterministic/Monte Carlo) radiation transport methods, codes, and applications at Oak Ridge National Laboratory, Proc. SNA+MC 2010, Tokyo, Japan, October 17-21, (2010). [CD-ROM] 